\title{
Toints
}

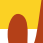

\section{Improving the accuracy of the preoperative diagnosis of long head of the biceps pathology: the biceps resisted flexion test}

\author{
PAOLO ARRIGONI ${ }^{1}$, VINCENZA RAGONE ${ }^{1}$, RICCARDO D'AMBROSI ${ }^{1}$, PATRICK DENARD², \\ FILIPPO RANDELLI ${ }^{1}$, GIUSEPPE BANFI ${ }^{3,4}$, PAOLO CABITZA ${ }^{1,3}$, PIETRO RANDELLI ${ }^{1,3}$ \\ ${ }^{1}$ IRCCS Policlinico San Donato Hospital, San Donato Milanese, Milan, Italy \\ 2 Southern Oregon Orthopaedics, Medford, OR, USA \\ ${ }^{3}$ Department of Biomedical Health Sciences, University of Milan, Milan, Italy \\ ${ }^{4}$ IRCCS Galeazzi Orthopaedic Institute, Milan, Italy
}

\begin{abstract}
Purpose: the purpose of this study was to describe a new test for identifying lesions of the long head of the biceps (LHB) and to evaluate its diagnostic performance in comparison with selected traditional clinical tests.

Methods: one hundred and nine consecutive candidates for arthroscopic rotator cuff repair were prospectively recruited.The BRF test, which measures biceps resisted flexion strength, was performed with the patient seated (armat the side and elbow flexed at $90^{\circ}$ ). The patient was asked to maintain maximal resistance for five seconds while strength was assessed with a digital dynamometer. Since the dynamometer expresses a continuous variable in kilograms, the measure was dichotomized using a threshold value (cut-off) valueable to simultaneously maximize the sensitivity and specificity. This cut-off value was derived from receiver operating characteristic (ROC) curve analysis.

Speed's test and the O'Brien test were also performed. During arthroscopy the presence of LHB pathology was assessed.
\end{abstract}

Results: biceps resisted flexion strength was significantly higher in patients without associated LHB lesions [median (range): $3 \mathrm{~kg}(0-9.5 \mathrm{~kg})$ versus $0 \mathrm{~kg}(0$ $8.5 \mathrm{~kg}$ ); $\mathrm{p}<0.001$ ]. The cut-off level able to simultaneously maximize the sensitivity and specificity of the test was $1.1 \mathrm{~kg}$.

Corresponding Author:

Paolo Arrigoni, MD

IRCCS Policlinico San Donato Hospital

Piazza Edmondo Malan 1, 20097 San Donato Milanese,

Milan, Italy

E-mail: arrigoni.p@gmail.com
The area under the ROC curve was $0.745(\mathrm{p}<0.001)$ for the dichotomic BRF test (dBRF), $0.562(\mathrm{p}=0.3)$ for the O'Brien test, and $0.602(\mathrm{p}=0.113)$ for Speed's test. A significant good level of accuracy was achieved only by the dBRF test. Specificity and the positive predictive value were significantly higher for the $\mathrm{dBRF}$ test than for Speed's and O'Brien's tests $(\mathrm{p}<0.02)$. Age and the dBRF test were both found to be significant predictors of LHB lesions.

Conclusions: the dBRF test showed higher accuracy than traditional clinical tests in diagnosing LHB lesions. This novel test for biceps pathology may be advantageous because it is objective and therefore likely reproducible.

Level of Evidence: Level II, Development of diagnostic test on basis of consecutive patients (with universally applied reference "gold" standard).

Key Words: arthroscopy, shoulder, biceps tendon, diagnostic, test.

\section{Introduction}

Disorders of the long head of the biceps tendon (LHB) are common and may be associated with abnormalities of other components of the rotator cuff (1). The clinical diagnosis of LHB lesions, however, is difficult (2). Most of the traditional clinical tests do not accurately identify biceps tendon lesions, showing relatively high sensitivity but poor specificity and a poor positive predictive value (3-5).

The reported accuracy of the commonly used Speed's and O'Brien tests is only 54 and $47 \%$ respectively (6). The purpose of this prospective evaluation was to evaluate the accuracy of the biceps resisted flexion test 
(BRF), a new test for diagnosing intra-articular LHB pathology, as compared with the traditional Speed's and O'Brien tests. Our hypothesis was that the BRF test would be more accurate than the traditional clinical tests in determining the presence of LHB lesions.

\section{Methods}

We performed a prospective preoperative evaluation of patients seen in a single institute over a period of six months. Patients undergoing arthroscopic rotator cuff repair were considered eligible for the study. Exclusion criteria were: the presence of unequivocally diagnosed concomitant disorders of the shoulder prior to surgery or during surgery, including glenohumeral arthritis, pseudoparalytic clinical presentations, fractures, osteonecrosis, labral pathology (including SLAP lesions), infection or prior surgery to the affected shoulder. One hundred and nine patients ( 52 females and 57 males) met the inclusion criteria and all agreed to participate. Their mean age at surgery was $60.6 \pm 10.6$ years.

Preoperatively, each patient was evaluated by an independent examiner who did not take part in the surgery. Three tests were performed: Speed's test, the O'Brien test, and the BRF test.

Speed's test was performed with the patient's shoulder flexed at $90^{\circ}$ in maximal supination with the elbow extended; the patient was then asked to resist a downward force applied to his/her palm. The test was considered positive when the patient reported pain (3). The O'Brien test was performed with the examiner positioned behind the patient. The patient was asked to maintain the shoulder at $90^{\circ}$ degrees of flexion and the arm in $10^{\circ}$ degrees of adduction (with respect to the sagittal plane) in full pronation (thumb down) while the examiner applied downward pressure. With the limb in the same position, but the hand fully supinated, the maneuver was repeated. The test was considered positive if pain was triggered by the first maneuver and decreased or disappeared with the second maneuver (5).

The BRF test was performed with the patient seated (arm at the side and elbow flexed at $90^{\circ}$ ). The patient was asked to maintain maximal resistance at $90^{\circ}$ of elbow flexion for five seconds (Fig. 1). A digital dynamometer (Kern \& Sohn GmbH, Balingen, Germany) linked to the ground was used to record

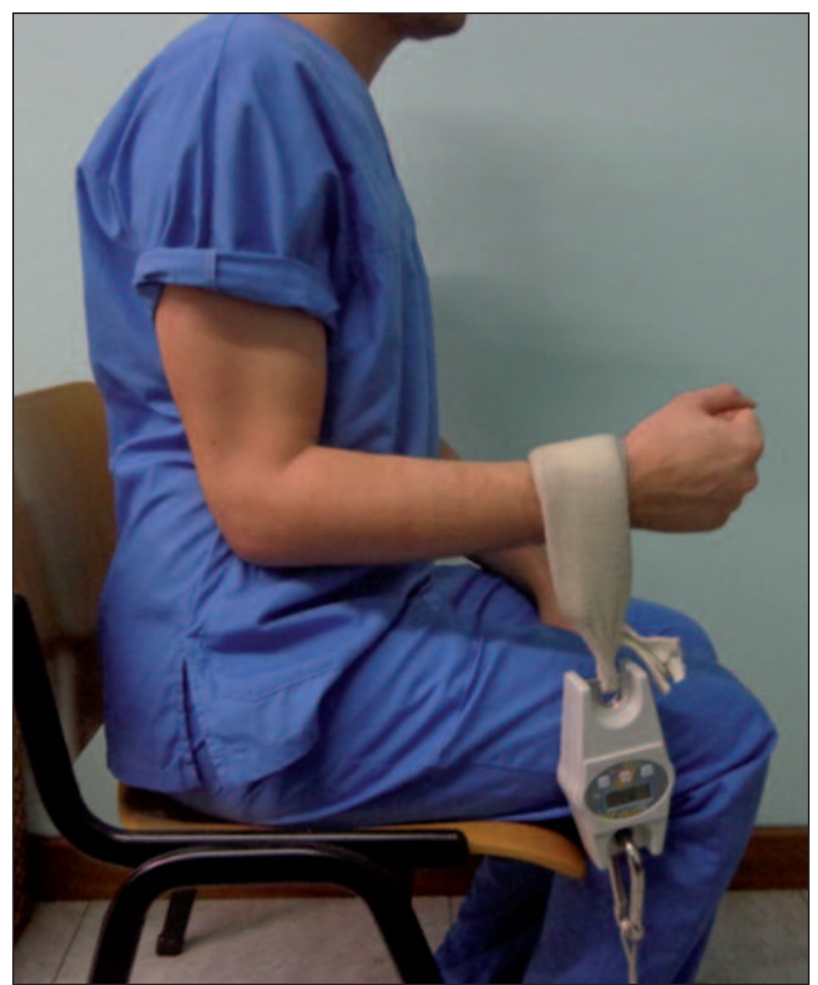

Fig. 1. The test is performed with the patient seated with the arm at the side, the elbow at $90^{\circ}$ of flexion and the forearm in neutral pronation/supination. The patient is asked to flex the affected limb against the resistance of a dynamometer and to maintain the maximal resistance for five seconds.

BRF strength. Pain during the week prior to surgery was also recorded using a visual analogue scale (VAS). All the procedures were performed on the same day as the clinical assessment by a single surgeon who was unaware of the results of clinical tests. Shoulder arthroscopy was performed in a lateral decubitus position.

During the diagnostic phase, the presence of LHB pathology was assessed via the posterior portaland classified as: spontaneous complete tear, intra-articular lesions (from $0.5 \mathrm{~cm}$ distal to the biceps anchor to 0.5 $\mathrm{cm}$ proximal to the biceps pulley), subclassified as less or more than $50 \%$ of tendon thickness, and pulley lesions. Pulley lesions were further subclassified as lesions involving the medial sling, the lateral sling, or complete lesions.

In the case of more than one type of LHB lesion, each lesion was considered an independent variable for assessment of the diagnostic performance of the clinical tests. 


\section{Statistical analysis}

Statistical analysis was performed by a trained statistician using dedicated statistical software (SPSS version 17, SPSS Inc., Chicago, USA). Continuous variables were reported as mean ( \pm standard deviation) or as median (range) values depending on the normal distribution of the data. Proportions were expressed for the nominal variables. The association between continuous variables and the presence of a lesion of the LHB was tested using ANOVA (one-way ANOVA) or the non-parametric Mann-Whitney test. Post-hoc comparisons between means were conducted using the Bonferroni test. For all the clinical tests, we calculated the number of true positives, the number of true negatives, the number of false positives, and the number of false negatives, to determine sensitivity, specificity, positive predictive value (PPV), and negative predictive value (NPV). The McNemar test was performed to compare these diagnostic values between the three clinical tests.

Since the dynamometer expresses a continuous variable in kilograms, the BRF test result was dichotomized using a threshold value (cut-off) value able to simultaneously maximize the sensitivity and specificity. The cut-off value was derived from receiver operating characteristic (ROC) curve analysis. ROC curves are graphic representations of sensitivity and specificity values and they were plotted for all the tests. The area under the ROC curve gives a measure of the test's performance (accuracy level); it ranges between 0.5 and 1.0. Conventionally accuracy is poor if the area is between 0.5 and 0.7 ; good between 0.7 and 0.9 ; excellent between 0.9 and 1 .

Chi-square tests were performed to determine whether the areas under the curves were greater than 0.50 (no discrimination) and to ascertain the relative accuracy of the diagnostic tests.

Variables were entered into a forward step wise logistic regression model to identify which of the clinical tests and the independent factors provide consistent predictions of LHB lesions.

A p-value less than 0.05 was considered significant for all tests.

\section{Results}

Of the 109 patients, $63(57.8 \%)$ had lesions involving the right shoulder. In 60 (55\%), the dominant arm was involved; $26 / 109$ patients (23.8\%) did not show any lesions of the biceps. The majority of patients had one LHB lesion (74 patients; 67.8\%), while nine $(8.2 \%)$ had two lesions. No patient had more than two lesions (Fig. 2).

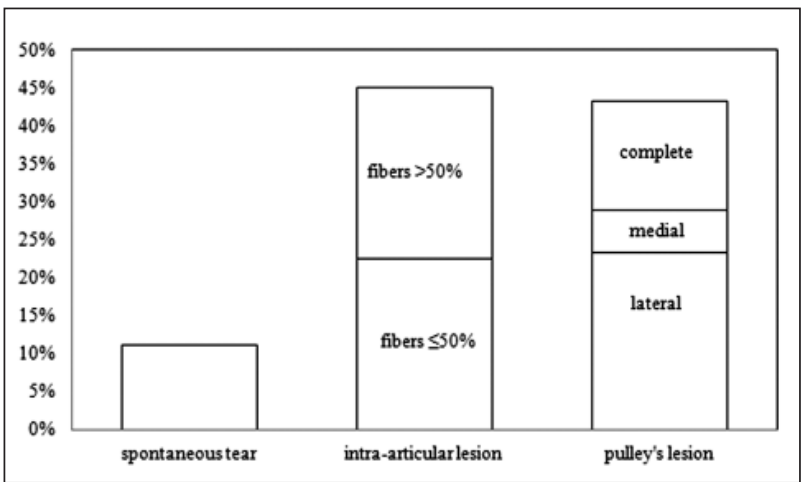

Fig. 2. Distribution of lesions of the long head of the biceps.

Patients presenting without LHB lesions were significantly younger $(50.7 \pm 10 \mathrm{yrs})$ than those with intra-articular lesions $(63.2 \pm 8.6 \mathrm{yrs})$, pulley lesions $(63 \pm 9.4 \mathrm{yrs})$ or spontaneous ruptures $(68.2 \pm 7.7)$ (p-value $<0.001)$.

No significant association was observed between pain (VAS) and the presence of LHB lesions $(6.3 \pm 2.5$ versus $6.2 \pm 2 ; \mathrm{p}>0.05$ ).

Biceps resisted flexion strength was significantly higher in patients without LHB lesions than in those with LHB lesions [median (range): $3 \mathrm{~kg}(0-9.5 \mathrm{~kg})$ versus 0 $\mathrm{kg}(0-8.5 \mathrm{~kg}) ; \mathrm{p}<0.001]$. In particular, strength was significantly lower in patients with intra-articular lesions [median (range): $0 \mathrm{~kg}(0-8.5 \mathrm{~kg}) ; \mathrm{p}<0.001]$ or pulley disorders [median (range): $0 \mathrm{~kg}(0-8 \mathrm{~kg})$; $\mathrm{p}=0.001]$. Within the pulley pattern group, strength was higher in patients with a lateral lesion than in those with a medial or complete lesion [median (range): 2.3 $\mathrm{kg}(0-8 \mathrm{~kg})$ versus $0 \mathrm{~kg}(0-2.5 \mathrm{~kg}) ; \mathrm{p}=0.01]$.

The cut-off level able to simultaneously maximize the sensitivity and specificity of the test, derived from the ROC curve, was $1.1 \mathrm{~kg}$.

Considering the BRF measure as a dichotomic variable, the test was considered negative if the measure was greater than $1.1 \mathrm{~kg}$ and the area under the ROC curve was $0.745(\mathrm{p}<0.001)$ for the dichotomic BRF (dBRF) test, $0.562(\mathrm{p}=0.3)$ for the O'Brien test, and 0.602 ( $\mathrm{p}=0.113)$ for Speed's test. A significant level of accuracy was achieved only by the dBRF test.

The specificity value and the PPV were significantly 
higher for the dBRF test than for the O'Brien test and Speed's test $(\mathrm{p}<0.02)$. The sensitivity value and NPV were significantly greater for Speed's test than for the O'Brien and dBRF tests $(\mathrm{p}<0.001)$ (Tab. 1).

Logistic regression analysis showed that age and the $\mathrm{dBRF}$ test were predictors of damage to the LHB. For each one year increase in age the risk of LHB lesion increased by a factor of $1.2(\mathrm{p}<0.001 ; 95 \%$ CI: 1.067 , 1.230). Patients with a positive dBRF test were 10.4 times more likely than patients with a negative test to show a lesion of the LHB ( $\mathrm{p}=0.004 ; 95 \%$ CI: 2.09 , 52.068). Speed's test and the O'Brien test were not predictive of LHB lesions ( $p>0.05$ ).

\section{Discussion}

Lesions of the LHB tendon are frequent in the adult population with are ported overall incidence of between 29 and $66 \%(4,7,8)$; they are associated with up to $90 \%$ of rotator cuff tears $(6,9)$. A $4 \%$ incidence of isolated LHB lesions has also been reported (10). Clinical trial shave shown that traditional tests do not accurately predict LHB lesions, and they therefore support the need for more precise preoperative clinical tests. The performance of Speed's test was first evaluated by Bennett (3), who reported sensitivity and specificity values of 90 and $13.8 \%$, respectively. Gillet al. (6) found sensitivity, specificity and accuracy values of 67,50 and 54\% respectively. The O'Brien test was also found to be limited, showing $68 \%$ sensitivity, $46 \%$ specificity, and $47 \%$ accuracy for partial lesions of the LHB (6). Other authors obtained lower sensitivity $(38 \%)$ but higher specificity and accuracy $(61 \%$ and $53 \%$ ) values (11). In the current study, the dBRF test showed a superior clinical performance in detecting detect LHB lesions.

The upper cut test for the biceps is another recently described test (11).This is performed with the involved shoulder in a neutral position, the elbow flexed at $90^{\circ}$, the forearm supinated, and the patient making a fist. The patient is asked to rapidly bring the hand up and towards the chin in an "upper cut" boxing-style punch. The examiner places his hand over the patient's fist and resists the motion. The test is positive if a painful pop over the anterior part of the shoulder is experienced. The BRF test measures strength in kilograms and therefore is objective. The upper cut test was found to show sensitivity and specificity values of 77 and $80 \%$, respectively, versus the 60 and $88 \%$ of the BRF.

Pain related to rotator cuff tears is exacerbated by abduction/elevation, biasing traditional tests which examine the biceps in forward flexion $(12,13)$. The BRF test is performed with the arm at the patient's side. This position reduces any pain due to the concomitant presence of rotator cuff tears $(12,13)$, and this probably improves the test performance. Since the BRF test result is objectively measured by a digital dynamometer there is less potential for subjective interpretation between examiners. Finally, the dichotomic version of the BRF test (dBRF), since it applies a threshold value, could be used for self-assessment of LHB pathology, asking the patient whether she/he feels pain while lifting a $1.1 \mathrm{~kg}$ (approx. $2.5 \mathrm{lb}$ ) weight. This possibility needs to be further analyzed to ascertain the reliability of such an application.

Although the power analysis shows $(1-\beta=0.8$ and $\alpha=$ $0.05)$ that the sample size is adequate to detect superiority of the dBRF over Speed's test and the O'Brien

Table 1. Diagnostic values of clinical tests for the LHB lesion.

\begin{tabular}{lccc}
\hline & Speed's Test & O'Brien Test & BRF \\
\hline Sensitivity (\%) & 93 & 66 & 60 \\
Specificity (\%) & 27 & 46 & 88 \\
PPV (\%) & 82 & 81 & 95 \\
NPV (\%) & 54 & 28 & 39 \\
Area under ROC curve (accuracy level) & 0.602 (poor) & 0.562 (poor) & 0.745 (good) \\
\hline
\end{tabular}

Abbreviations: $\mathrm{BRF}=$ biceps resisted flexion; $\mathrm{PPV}=$ positive predictive value; $\mathrm{NPV}=$ negative predictive value; ROC=receiver operating characteristic 
tests in terms of accuracy and specificity, further studies with a large sample size are necessary to validate this finding. Since the choice of cut-off value is the key point for the wide spread use of this test in current clinical practice, more studies evaluating its intraobserver reliability are needed.

Our data also demonstrate that pain (VAS) reported by patients in the seven days before surgery is not associated with the presence of LHB lesions, highlighting the need to investigate all the other associated shoulder diseases.

The mean age was significantly lower in the patients who did not have a biceps lesion. Similarly, Braun et al. (9) observed a mean age of 48.5 years in patients without injury to the pulley versus 57 years $(\mathrm{p}<0.001)$ in the pulley lesion group. Gill et al. (6) observed a significant difference in age between patients with and without intra-articular lesions (59 years versus 44 years; $p<0.0001)$. Age is a risk factor for these lesions, therefore in patients older than 65 years with concomitant tears of the rotator cuff it is prudent to anticipate a lesion of the LHB, which will be present in the majority of cases.

A limitation of this study, shared by most of the previous clinical studies investigating the performance of clinical tests in this field, is the presence of concomitant diseases of the rotator cuff. A stratified analysis for the presence of concomitant cuff tears should be conducted to determine the influence of these tears on the results. On the other hand, the condition investigated in this study is more representative of what tends to be observed in the real clinical setting, in which pre-diagnosing a biceps problem concomitant to a cuff tear can modify the surgical plan. Exclusion of SLAP lesions can might also be considered a limitation. Exploring the test performance to diagnose biceps root pathology will be a future research direction. The possible differences generated by pronation/supination of the hand also need to be analyzed.

In conclusion, the dBRF test is more accurate than traditional clinical tests in diagnosing LHB lesions. This novel test is objective and can be easily performed in the clinical setting.

\section{References}

1. Chen $\mathrm{CH}, \mathrm{Hsu} \mathrm{KY}$, Chen WJ, Shih CH. Incidence and severity of biceps long head tendon lesion in patients with complete rotator cuff tears. J Trauma. 2005;58:1189-1193.

2. Ahrens PM, Boileau P. The long head of biceps and associated tendinopathy. J Bone Joint Surg Br. 2007; 89:1001-1009.

3. Bennett WF. Specificity of the Speed's test: arthroscopic technique for evaluating the biceps tendon at the level of the bicipital groove. Arthroscopy. 1998;14:789-796.

4. Holtby R, Razmjou H. Accuracy of the Speed's and Yergason's tests in detecting biceps pathology and SLAP lesions: comparison with arthroscopic findings. Arthroscopy. 2004;20:231-236.

5. O'Brien SJ, Pagnani MJ, Fealy S, McGlynn SR, Wilson JB. The active compression test: a new and effective test for diagnosing labral tears and acromioclavicular joint abnormality. Am J Sports Med. 1998;26:610-613.

6. Gill HS, El Rassi G, Bahk MS, Castillo RC, McFarland EG. Physical examination for partial tears of the biceps tendon. Am J Sports Med. 2007;35:1334-1340.

7. Skendzel JG, Jacobson JA, Carpenter JE, Miller BS. Long head of biceps brachii tendon evaluation: accuracy of preoperative ultrasound. AJR Am J Roentgenol. 2011;197:942948.

8. Mohtadi NG, Vellet AD, Clark ML, et al. A prospective, double-blind comparison of magnetic resonance imaging and arthroscopy in the evaluation of patients presenting with shoulder pain. J Shoulder Elbow Surg. 2004;13:258-265.

9. Braun S, Horan MP, Elser F, Millett PJ. Lesions of the biceps pulley. Am J Sports Med. 2011;39:790-795.

10. Delle Rose G, Borroni M, Silvestro A, et al. The long head of biceps as a source of pain in active population: tenotomy or tenodesis? A comparison of 2 case series with isolated lesions. Musculoskelet Surg. 2012; 96 Suppl 1:S47-52.

11. Ben Kibler W, Sciascia AD, Hester P, Dome D, Jacobs C. Clinical utility of traditional and new tests in the diagnosis of biceps tendon injuries and superior labrum anterior and posterior lesions in the shoulder. Am J Sports Med. 2009;37:1840-1847.

12. Barrett WP, Franklin JL, Jackins SE, Wyss CR, Matsen FA 3rd. Total shoulder arthroplasty. J Bone Joint Surg Am. 1987;69:865-872.

13. Saha AK. The classic. Mechanism of shoulder movements and a plea for the recognition of "zeroposition" of glenohumeral joint.Clin Orthop Relat Res. 1983;(173):3-10. Relat Res. 1983;(173):3-10. 